ISSN: 2521-5051 (Print)

ISSN: 2521-506X (Online) CODEN : ASMCCQ

\title{
FIVE SEPARATION AXIOMS IN QUAD SOFT NON-LINEAR STRUCTURE
}

\author{
Fahad Jamal $^{3}$, Arif Mehmood Khattak ${ }^{1}$, Gulzar Ali Khan ${ }^{3}$, Saleem Abdullah ${ }^{2}$ \\ ${ }^{1}$ Department of Mathematics and Statistics, Riphah International University Islamabad, Pakistan. \\ ${ }^{2}$ Department of Mathematics, Abdul Wali Khan University Mardan, Pakistan. \\ ${ }^{3}$ Department of Mathematics, Qurtuba University Peshawar, Pakistan. \\ *Corresponding Author Email: mehdaniyal@gmail.com
}

This is an open access article distributed under the Creative Commons Attribution License, which permits unrestricted use, distribution, and reproduction in any medium, provided the original work is properly cited

\section{ARTICLE DETAILS}

\section{Article History:}

Received 12 November 2017 Accepted 12 December 2017 Available online 1 January 2018

\section{ABSTRACT}

In 1999, Russian mathematician Molodtsov planted the novel notion of a soft set which can be supposed as a new mathematical approach for imprecision. Topological structures of soft set have been studied by some mathematicians in current years. M. Shabir and M. Naz presented soft topological spaces and studied some key properties of them. Soft Quad topological spaces are nothing but just Extension of soft topological spaces. When with soft single topology three more soft topologies are hugged together through mathematical discipline then the notion of soft Quad topology will automatically come in play. The central objective of this article is to publicize soft separations axioms in soft quad topological spaces. Moreover to discuss the linkage among them through two paths.

KEYWORDS

Soft quad topological spaces soft $q T_{0}$ space, soft $q T_{1}$ space, soft $q T_{2}$ space, soft $q T_{3}$ and soft $q T_{4}$ space.

\section{INTRODUCTION}

Many practical problems in economics, engineering, environmental sciences, social sciences, medical sciences etc. cannot be dealt by classical methods because classical methods have inherent difficulties. The reason for these difficulties may be due to the insufficiency of the theories of parameterization tools. Numerous theories exist, for example, fuzzy set theory, intuitionistic fuzzy set theory, rough set theory, i.e., which can be considered as a mathematical tool for dealing with uncertainties [1-3]. Each of these theories has its inherent difficulties as what were pointed out by Molodtsov [4]. Molodtsov originated absolutely a new methodology for modelling uncertainties and applied successfully in directions such as smoothness of functions, game theory, operations research, Riemannintegration, Perron integration, and so on [4]. A group researcher gave the first practical application of soft sets in decision making problems [5]. The algebraic structure of set theories dealing with uncertainty is an important problem. Many researchers have contributed towards the algebraic structure of soft set theory. Some of researchers defined soft groups and deduced their fundamental characterizations [6]. Others group of researchers introduced initial concepts of soft rings [7]. Besides, in other study, researchers defined soft semi rings and several related notions to establish a connection between soft sets and semi rings [8]. Some researchers defined soft modules and investigated their basic properties [9]. A studied soft ideal over a semi groups which characterized generalized fuzzy ideals and fuzzy ideals with thresholds of a semi groups [10]. There are two researchers introduced fuzzy soft modules and intuitionistic fuzzy soft modules and investigated some basic properties $[11,12]$.

Shabir and Naz firstly introduced the notion of soft topological space which are defined over an initial universe with a fixed set of parameters and showed that a soft topological space gives a parameterized family of topological spaces [13]. Theoretical studies of soft topological spaces have also been by some authors in [14-17]. In these studies the concept of soft point is given almost samely. Consequently, some results of classical topology are not valid in soft topological spaces. A group researcher introduced the concepts of supra topological spaces, supra closed sets, supra open sets and supra continuous mapping [18]. Many results of topological spaces remain valid in supra topological spaces, but some become false. As a generalized of soft topological spaces, the notion of supra soft topological spaces by dropping only the soft intersection condition [19]. They extend some types of different kinds of subsets of soft topological spaces using the notion of $\gamma$-operation to supra soft topological space and study the decompositions of some forms of supra soft continuity. The notion of soft bi topological space which is defined over an initial universal set $\mathrm{X}$ with fixed set of parameters and studied some types of soft separation axioms [20]. It is clear that a soft bi topological space is a generalization of a soft topological space as every soft bi topological space $\left(X, \eta_{1}, \eta_{2}, E\right)$ if $\eta_{1}=\eta_{2}=\eta$ A. Generalized the notions of soft topological spaces such as open soft sets, closed soft sets, soft interior, and soft closure in a soft bi topological space [13]. Thus, they introduced the notions of pairwise open (closed) soft sets, pairwise soft interior operator in a soft bi topological space $\left(X, \eta_{1}, \eta_{2}, E\right)$ and showed that the family of all pairwise open soft sets is a supra soft topology which is containing $\eta_{1}, \eta_{2}$ but it is not soft topology [13].

In the present study, we first give some basic ideas about soft sets and the results already studied. In addition to these, we introduce the concept of soft point to the theory of soft set. According to this definition, we investigate some important notions of soft topological spaces. Later we give separability axioms in soft Quad topological spaces and study some of their characterizations. The separability axioms are the soft separation axioms in Soft Quad topological spaces with respect to ordinary and soft points.

We expect that these results will be of greater importance for the forthcoming learning on Soft Quad topological spaces to accomplish general framework for the most practical applications and to answer the most intricate problems containing doubt in economics, engineering, medical, environment and in general.

\section{PRELIMINARIES}

In this section we will introduce necessary definitions and theorems for soft sets. Molodtsov defined the soft set in the following way. Let $X$ be an initial universe set and $E$ be a set of parameters. Let $P(X)$ denotes the power set of $X[14]$.

Definition 2.1. ([4]) A pair $(F, E)$ is called a soft set over $X$; where $F$ is a mapping given by

$F: E \rightarrow P(X)$.

In other words, the soft set is a parameterized family of subsets of the set $\mathrm{X}$. For $e \in E ; F(e)$ may be considered as the set of $e$-elements of the soft 
$\operatorname{set}(F ; E)$; or as the set of $e$-approximate elements of the soft set, i.e. $(F, E)=\{(e, F(e)): e \in E, F: E \rightarrow P(X)\}$.

After this, $S S(X) E_{E}$ denotes the family of all soft sets over $X$ with a fixed set of parameter $E$.

Definition 2.2. ([21]) For two soft sets $(F, E)$ and $(G, E)$ over $X,(F, E)$ is called a soft subset of $(G, E)$ if $\forall e \in E, F(e) \subseteq G(e)$. This relationship is denoted by $(F, E) \widetilde{\widetilde{\subseteq}}(G, E)$.

Similarly, $(F, E)$ is called a soft superset of $(G, E)$ if $(G, E)$ is a soft subset of $(F, E)$. This

Linkage is denoted by $(F, E) \widetilde{\Xi}(G, E)$. Two soft sets $(F, E)$ and $(G, E)$ over $X$ are called soft equal if $(F, E)$ is a soft subset of $(G, E)$ and $(G, E)$ is a soft subset of $(F, E)$.

Definition 2.3. ([21])The intersection of two soft sets $(F, E)$ and $(G, E)$ over $X$ is the soft set

$(H, E)$, where $\forall e \in E, \quad H(e)=F(e) \cap G(e)$. This is denoted $\operatorname{by}(F, E) \widetilde{\widetilde{n}}(G, E)=(H, E)$.

Definition 2.4. ([21]) the union of two soft sets $(F, E)$ and $(G, E)$ over $X$ is the soft set $(H, E)$,

Where $\forall e \in E, H(e)=F(e) \cup G(e)$ This is denoted by $(F, E) \widetilde{\widetilde{U}}(G, E)=$ $(H, E)$.

Definition 2.5. ([22]) A soft set $(F, E)$ over $X$ is said to be a null soft set denoted by $\varphi$ if for alle $\in E, F(e)=\varphi$.

Definition 2.6. ([22]) A soft set $(F, E)$ over $X$ is said to be an absolute soft set denoted by $\tilde{X}$

If for alle $\in E ; F(e)=X$.

Definition 2.7. ([23]) The soft set $(F, A) \in S S(X)_{A}$ is called a soft point in $X_{A}$, denoted by $e_{F}$, if for the element $e \in A, F(e) \neq \emptyset$ and $F\left(e^{\prime}\right)=\phi$ if for all $e^{\prime} \in A-\{e\}$

Definition 2.8. ([22]) the complement of a soft set $(F, E)$; denoted by $(F, E)^{c}$, is defined as $(F, E)^{c}=\left(F^{c} ; E\right)$; where $F^{c}: E \rightarrow P(X)$ is a mapping given $\operatorname{by} F^{c}(e)=X \backslash F(e) ; \forall e \in E$ and $F^{c}$ is called the soft complement function of $\mathrm{F}$ :

Definition 2.9. ([22]) Let $Y$ be a non-empty subset of $X$, then $\tilde{Y}$ denotes the soft set $(Y, E)$ over $X$ for which $(e)=Y$, for all $\in E$.

Definition 2.10. ([22]) Let $(F, E)$ be a soft set over $X$ and $Y$ be a nonempty subset of $X$. Then

The sub soft set of $(F, E)$ over $Y$ denoted by $\left({ }^{Y} F, E\right)$, is defined as follows ${ }^{\mathrm{Y}} F(e)=Y \cap F(e)$, for all $\forall e \in E$

In other words $\left({ }^{Y} F, E\right)=\tilde{\tilde{Y}} \cap F(e)$

Definition 2.11. ([22]) Let $\mathfrak{\Im}$ be the collection of soft sets over X, then $\mathfrak{J}$ is said to be a soft

Topology on X if

(1) $\varphi, \tilde{\tilde{X}} \in \mathfrak{I}$

(2) The union of any number of soft sets in $\mathfrak{I}$ belongs to $\mathfrak{I}$

(3) The intersection of any two soft sets in $\Im$ belongs to $\mathfrak{J}$

The triplet $(X, \mathfrak{I}, E)$ is called a soft topological space over $X$.

Proposition 2.12. ([22]) Let $(X, \mathfrak{J}, E)$ be a soft topological space over $X$. Then the collection

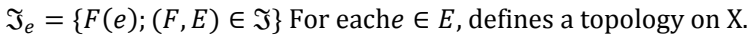

Definition 2.13. ([22]) Let $(X, \mathfrak{J}, E)$ be a soft topological space over $X$ and $(F, E)$ be a soft set over. Then the soft closure of $(F, E)$, denoted by $\overline{\overline{(F, E)}}$ is the intersection of all soft closed super sets of $(F, E)$. Clearly $\overline{\overline{(F, E)}}$ is the smallest soft closed set over $X$ which contains $(F, E)$.

Proposition 1. ([22]) Let $(X, \tau, E)$ be a soft topological space over $X$.If $(X, \tau, E)$ is soft $T_{3}$-space, then for all $x \in X, x_{E}=(x, E)$ is-closed soft set.

Proposition 2. ([22]) Let $\left(Y, \tau_{Y, E)}\right.$ be a soft sub space of a soft topological space $(X, \tau, E)$ and $(F, E) \in S S(X)$ then

1. If $(F, E)$ is soft open set in $Y$ and $Y \in \tau$, then $(F, E) \in \tau$

2. $(F, E)$ is soft open soft set in $Y$ if and only if $(F, E)=Y \cap(G, E)$ for some $(G, E) \in \tau$.

3. $(F, E)$ is soft closed soft set in $Y$ if and only if $(F, E)=Y \cap(H, E)$ for some $(H, E)$ is $\tau$ soft closed.
3. SOME BASIC RESULTS IN QUAD SOFT TOPOLOGY WITH RESPECT TO CRISP POINTS

Definition 3.1. Let $\left(X, \tau_{1}, \tau_{2}, \tau_{3}, \tau_{4}, E\right)$ be a quad soft topological space over $X$, where $\left(X, \tau_{1}, E\right),\left(X, \tau_{2}, E\right),\left(X, \tau_{3}, E\right)$ and $\left(X, \tau_{4}, E\right)$ be four different soft topologies on $X$. Then a sub set $(F, E)$ is said to be quad-open (in short hand q-open) if $(F, E) \subseteq \tau_{1} \cup \tau_{2} \cup \tau_{3} \cup \tau_{4}$ and its complement is said to be soft q-closed.

Definition 3.2. Let $\left(X, \tau_{1}, \tau_{2}, \tau_{3}, \tau_{4}, E\right)$ be a quad soft topological space over $X,(G, E)$ be a soft set over $X$ and $x \in X$. Then $x$ is said to be a soft $\mathrm{q}$ interior point of $(G, E)$ if there exists soft q-open set $(F, E)$ such that $x \in$ $(F, E) \subseteq(G, E)$.

Definition 3.3. Let $\left(X, \tau_{1}, \tau_{2}, \tau_{3}, \tau_{4}, E\right)$ be a soft quad topological space over $X,(G, E)$ be a soft set over $X$ and $x \in X$. Then $(G, E)$ is said to be a soft q neighborhood of $x$ if there exists soft q-open set $(F, E)$ such that $x \in$ $(F, E) \subseteq(G, E)$

Proposition 3. Let $\left(X, \tau_{1}, \tau_{2}, \tau_{3}, \tau_{4}, E\right)$ be a soft quad topological space over $X,(G, E)$ be a soft set over $X$ and $x \in X$. If $\mathrm{x}$ is a soft interior point of $(G, E)$ then $x$ is an interior point of $G(\alpha) \operatorname{in}\left(\tau_{1} \cup \tau_{2} \cup \tau_{3} \cup \tau_{4}\right)_{(\alpha)}$ for each $\alpha \in E$.

Proof. For any $\alpha \in E, G(\alpha) \subseteq X$. If $x \in X$ is a soft interior point of $(G, E)$ then there exists soft q-open set $(F, E)$ such that $x \in(F, E) \subseteq(G, E)$. This means that $x \in F(\alpha) \subseteq G(\alpha)$. As $F(\alpha) \in\left(X, \tau_{1}, \tau_{2}, \tau_{3}, \tau_{4}, E\right)$ so $F(\alpha)$ is open set in $\left(\tau_{1} \cup \tau_{2} \cup \tau_{3} \cup \tau_{4}\right)_{(\alpha)}$ and $x \in F(\alpha)$. This implies that $\mathrm{x}$ is an interior point of $G(\alpha) \operatorname{in}\left(\tau_{1} \cup \tau_{2} \cup \tau_{3} \cup \tau_{4}\right)_{(\alpha)}$

Proposition 4. Let $\left(X, \tau_{1}, \tau_{2}, \tau_{3}, \tau_{4}, E\right)$ be a soft quad topological space over $X$, then each $x \in X$ has a soft q neighborhood;

If $(F, E)$ and $(G, E)$ are soft q neighborhoods of somex $\in X$, then $(F, E) \cap$ $(G, E)$ is also a soft neighborhood of $x$.

If $(F, E)$ is a soft q neighborhood of $x \in X$ and $(F, E) \subseteq(G, E)$, then $(G, E)$ is also a soft q neighborhood of $x \in X$.

Proof. (1) For any $x \in X, \mathrm{x} \in \tilde{X}$ and since $\tilde{X} \in\left(\tau_{1} \cup \tau_{2} \cup \tau_{3} \cup \tau_{4}\right)$, so $x \in$ $X \subseteq \tilde{X}$.

Thus $\tilde{X}$ is a soft neighborhood of $x$.

Let $(F, E)$ and $(G, E)$ be the soft neighborhoods of $x \in X$, then there exist soft q open sets $\left(F_{1}, E\right),\left(F_{2}, E\right) \in\left(\tau_{1} \cup \tau_{2} \cup \tau_{3} \cup \tau_{4}\right)$ such that

$$
x \in\left(F_{1}, E\right) \subseteq(F, E) \text {. And } x \in\left(F_{2}, E\right) \subseteq(G, E)
$$

Now $x \in\left(F_{1}, E\right)$ and $x \in\left(F_{2}, E\right)$ implies that $\left(F_{1}, E\right) \cap$ $\left(F_{2}, E\right)$ and $\left(F_{1}, E\right) \cap\left(F_{2}, E\right) \in\left(\tau_{1} \cup \tau_{2} \cup \tau_{3} \cup \tau_{4}\right)$. So we have $x \in\left(F_{1}, E\right) \cap\left(F_{2}, E\right) \subseteq(F, E) \cap(G, E)$

Thus $(F, E) \cap(G, E)$ is a soft neighborhood of $x$.

Let $(F, E)$ be a soft q neighborhood of $x \in X$ and $(F, E) \subseteq(G, E)$. By definition there exists a soft q open set $\left(F_{1}, E\right)$ such that $x \in\left(F_{1}, E\right) \subseteq$ $(F, E) \subseteq(G, E)$.

$$
\text { Thus } x \in\left(F_{1}, E\right) \subseteq(G, E)
$$

Hence $(G, E)$ is a soft q neighborhood of $x$.

Proposition 5. Let $\left(X, \tau_{1}, \tau_{2}, \tau_{3}, \tau_{4}, E\right)$ be a soft quad topological space over $X$.Then $\left(X, \tau_{1}, \tau_{2}, \tau_{3}, \tau_{4}, E\right)$ is soft $q-T_{1}$ space iff each soft point is a soft q closed set.

Proof. Let $\left(X, \tau_{1}, \tau_{2}, \tau_{3}, \tau_{4}, E\right)$ is soft $T_{1}$ space and $(x, E)$ be an arbitrary soft point. We show that $(x, E){ }^{c}$ is a soft q open set. Let $(y, E) \in(x, E){ }^{c}$ such that $(x, E) \neq(y, E)$. Since $\left(X, \tau_{1}, \tau_{2}, \tau_{3}, \tau_{4}, E\right)$ is soft $T_{1}$ space, there exists q soft open set $(G, E)$ such that $(y, E) \in(G, E),(x, E) \notin(G, E)$.Then $(y, E) \in$ $(G, E) \subseteq(x, E)^{c}$.This implies that $(x, E)^{c}$ is a $\mathrm{q}$ soft open set. Equivalently, $(x, E)$ is a q soft closed set. Suppose that for each $(x, E)$ is a $\mathrm{q}$ soft closed set. Then automatically $(x, E)^{c}$ is a q $\operatorname{soft}$ open $\operatorname{set} \operatorname{Let}(x, E) \neq$ $(y, E)$. Thus $(y, E) \in\left(x_{e}, E\right)^{c}$ and $(x, E) \in(x, E)^{c}$. In a similar fashion $(y, E){ }^{c}$ is a q soft open set such that $(x, E) \in(y, E){ }^{c} \cdot \operatorname{and}(y, E) \notin(y, E)^{c}$. Therefore $\left(X, \tau_{1}, \tau_{2}, \tau_{3}, \tau_{4}, E\right)$ is a soft $q-T_{1}$ space

\section{SOME BASIC RESULTS IN QUAD SOFT TOPOLOGY WITH RESPECT TO SOFT POINTS}

Definition 4.1. Let $\left(X, \tau_{1}, \tau_{2}, \tau_{3}, \tau_{4}, E\right)$ be a quad soft topological space over $X$, where $\left(X, \tau_{1}, E\right),\left(X, \tau_{2}, E\right),\left(X, \tau_{3}, E\right)$ and $\left(X, \tau_{4}, E\right)$ be four different soft topologies on $X$. Then a sub set $(F, E)$ is said to be quad-open (in short 
hand q-open) if $(F, E) \subseteq \tau_{1} \cup \tau_{2} \cup \tau_{3} \cup \tau_{4}$ and its complement is said to be soft q-closed.

Definition 4.2. Let $\left(X, \tau_{1}, \tau_{2}, \tau_{3}, \tau_{4}, E\right)$ be a quad soft topological space over $X,(G, E)$ be a soft set over $X$ and $x_{e} \in X$. Then $x$ is said to be a soft $\mathrm{q}$ interior point of $(G, E)$ if there exists soft q-open set $(F, E)$ such that $x_{e} \in$ $(F, E) \subseteq(G, E)$.

Definition 4.3. Let $\left(X, \tau_{1}, \tau_{2}, \tau_{3}, \tau_{4}, E\right)$ be a soft quad topological space over $X,(G, E)$ be a soft set over $X$ and $x_{e} \in X$. Then $(G, E)$ is said to be a soft q neighborhood of $x$ if there exists soft q-open set $(F, E)$ such that $x_{e} \in$ $(F, E) \subseteq(G, E)$

Proposition 6. Let $\left(X, \tau_{1}, \tau_{2}, \tau_{3}, \tau_{4}, E\right)$ be a soft quad topological space over $X,(G, E)$ be a soft set over $X$ and $x_{e} \in X$. If $\mathrm{x}$ is a soft interior point of $(G, E)$ then $x$ is an interior point of $G(\alpha) \operatorname{in}\left(\tau_{1} \cup \tau_{2} \cup \tau_{3} \cup \tau_{4}\right)_{(\alpha)}$ for each $\alpha \in E$.

Proof. For any $\alpha \in E, G(\alpha) \subseteq X$. If $x \in X$ is a soft interior point of $(G, E)$ then there exists soft q-open set $(F, E)$ such that $x \in(F, E) \subseteq(G, E)$. This means that $x \in F(\alpha) \subseteq G(\alpha)$. As $F(\alpha) \in\left(X, \tau_{1}, \tau_{2}, \tau_{3}, \tau_{4}, E\right)$ so $F(\alpha)$ is open set in $\left(\tau_{1} \cup \tau_{2} \cup \tau_{3} \cup \tau_{4}\right)_{(\alpha)}$ and $x \in F(\alpha)$. This implies that $\mathrm{x}$ is an interior point of $G(\alpha) \operatorname{in}\left(\tau_{1} \cup \tau_{2} \cup \tau_{3} \cup \tau_{4}\right)_{(\alpha)}$

Proposition 7. Let $\left(X, \tau_{1}, \tau_{2}, \tau_{3}, \tau_{4}, E\right)$ be a soft quad topological space over $X$, then each $x_{e} \in X$ has a soft q neighborhood;

If $(F, E)$ and $(G, E)$ are soft q neighborhoods of some $x_{e} \in X$, then $(F, E) \cap$ $(G, E)$ is also a soft neighborhood of $x$

If $(F, E)$ is a soft q neighborhood of $x_{e} \in X$ and $(F, E) \subseteq(G, E)$, then $(G, E)$ is also a soft q neighborhood of $x_{e} \in X$.

Proof. (1) For any $x_{e} \in X, x_{e} \in \tilde{X}$ and since $\tilde{X} \in\left(\tau_{1} \cup \tau_{2} \cup \tau_{3} \cup \tau_{4}\right)$, so $x_{e} \in$ $X \subseteq \tilde{X}$.

Thus $\tilde{X}$ is a soft neighborhood of $x_{e}$.

Let $(F, E)$ and $(G, E)$ be the soft neighborhoods of $x_{e} \in X$, then there exist soft q open sets $\left(F_{1}, E\right),\left(F_{2}, E\right) \in\left(\tau_{1} \cup \tau_{2} \cup \tau_{3} \cup \tau_{4}\right)$ such that

$$
x_{e} \in\left(F_{1}, E\right) \subseteq(F, E) \text { And } x_{e} \in\left(F_{2}, E\right) \subseteq(G, E)
$$

Now $x_{e} \in\left(F_{1}, E\right)$ and $x_{e} \in\left(F_{2}, E\right)$ implies that $\left(F_{1}, E\right) \cap$ $\left(F_{2}, E\right)$ and $\left(F_{1}, E\right) \cap\left(F_{2}, E\right) \in\left(\tau_{1} \cup \tau_{2} \cup \tau_{3} \cup \tau_{4}\right)$. So we have $x_{e} \in\left(F_{1}, E\right) \cap\left(F_{2}, E\right) \subseteq(F, E) \cap(G, E)$.

Thus $(F, E) \cap(G, E)$ is a soft neighborhood of $x$. Let $(F, E)$ be a soft q neighborhood of $x_{e} \in X$ and $(F, E) \subseteq(G, E)$.So by definition there exists a soft q open set $\left(F_{1}, E\right)$ such that $x_{e} \in\left(F_{1}, E\right) \subseteq$ $(F, E) \subseteq(G, E)$.

$$
\text { Thus } x_{e} \in\left(F_{1}, E\right) \subseteq(G, E)
$$

Hence $(G, E)$ is a soft q neighborhood of $x_{e}$.

Proposition 8. Let $\left(X, \tau_{1}, \tau_{2}, \tau_{3}, \tau_{4}, E\right)$ be a soft quad topological space overX.Then $\left(X, \tau_{1}, \tau_{2}, \tau_{3}, \tau_{4}, E\right)$ is soft $q-T_{1}$ space iff each soft point is a soft q closed set.

Proof. Let $\left(X, \tau_{1}, \tau_{2}, \tau_{3}, \tau_{4}, E\right)$ is soft $T_{1}$ space and $\left(x_{e}, E\right)$ be an arbitrary soft point. We show that $\left(x_{e}, E\right)^{c}$ is a soft q open set. Let $\left(y_{e^{\prime}}, E\right) \in$ $\left(x_{e}, E\right)^{c}$ such that $\left(x_{e}, E\right) \neq\left(y_{e^{\prime}}, E\right)$. Since $\left(X, \tau_{1}, \tau_{2}, \tau_{3}, \tau_{4}, E\right)$ is soft $T_{1}$ space, there exists q soft open set $(G, E)$ such that $\left(y_{e^{\prime}}, E\right) \in(G, E)$, $\left(x_{e}, E\right) \notin(G, E)$.Then $\left(y_{e^{\prime}}, E\right) \in(G, E) \subseteq\left(x_{e}, E\right)^{c}$.This implies that $\left(x_{e}, E\right){ }^{c}$ is a q soft open set. Equivalently, $\left(x_{e}, E\right)$ is a q soft closed set. Suppose that for each $\left(x_{e}, E\right)$ is a q soft closed set. Then automatically $\left(x_{e}, E\right)^{c}$ is a q soft open set. Let $\left(x_{e}, E\right) \neq\left(y_{e^{\prime}}, E\right)$. Thus $\left(y_{e^{\prime}}, E\right) \in\left(x_{e}, E\right)^{c}$ and $\left(x_{e}, E\right) \in\left(x_{e}, E\right)^{c}$. In a similar fashion $\left(y_{\left.e^{\prime}, E\right)}{ }^{c}\right.$ is a q soft open set such that $\left(x_{e}, E\right) \in\left(y_{e^{\prime}}, E\right)^{c}$.and $\left(y_{e^{\prime}}, E\right) \notin\left(y_{e^{\prime}}, E\right){ }^{c}$. Therefore $\left(X, \tau_{1}, \tau_{2}, \tau_{3}, \tau_{4}, E\right)$ is a soft $q-T_{1}$ space

\section{SOFT SEPARATION AXIOMS IN QUAD SOFT TOPOLGICAL SPACES}

In this section we inaugurated soft separation axioms in soft quad topological space with respect to ordinary points and discussed some results with respect to these points in detail.

Definition 5.1. Let $\left(X, \tau_{1}, E\right),\left(X, \tau_{2}, E\right),\left(X, \tau_{3}, E\right)$ and $\left(X, \tau_{4}, E\right)$ be four different soft topologies on $X$. Then $\left(X, \tau_{1}, \tau_{2}, \tau_{3}, \tau_{4}, E\right)$ is called a soft quad topological space. The soft four topologies $\left(X, \tau_{1}, E\right)$, $\left(X, \tau_{2}, E\right),\left(X, \tau_{3}, E\right)$ and $\left(X, \tau_{4}, E\right)$ are independently satisfying the axioms of soft topology. The members of $\tau_{1}$ are called $\tau_{1}$ soft open set. And complement of $\tau_{1}$ Soft open set is called $\tau_{1}$ soft closed set. Similarly, the member of $\tau_{2}$ are called $\tau_{2}$ soft open sets and the complement of $\tau_{2}$ soft open sets are called $\tau_{2}$ soft closed set. The members of $\tau_{3}$ are called $\tau_{3}$ soft open set. And complement of $\tau_{3}$ Soft open set is called $\tau_{3}$ soft closed set and the members of $\tau_{4}$ are called $\tau_{4}$ soft open set. And complement of $\tau_{4}$ Soft open set is called $\tau_{4}$ soft closed set.

Definition 5.2. Let $\left(X, \tau_{1}, \tau_{2}, \tau_{3}, \tau_{4}, E\right)$ be a soft quad topological space over $X$ and $x, y \in X$ such that $x \neq y$

If we can find soft q-open sets $(F, E)$ and $(G, E)$ such that $x \in(F, E)$ and $y \notin(F, E)$ or $y \in(G, E)$ and $x \notin(G, E)$ then $\left(X, \tau_{1}, \tau_{2}, \tau_{3}, \tau_{4}, E\right)$ is called soft $q T_{0}$ space.

Definition 5.3. Let $\left(X, \tau_{1}, \tau_{2}, \tau_{3}, \tau_{4}, E\right)$ be a soft quad topological space over $\mathrm{X}$ and $x, y \in X$ such that $x \neq y$ If we can find two soft q-open sets $(F, E)$ and $(G, E)$ such that $x \in(F, E)$ and $y \notin(F, E)$ and $y \in(G, E)$ and $x \notin(G, E)$ then $\left(X, \tau_{1}, \tau_{2}, \tau_{3}, \tau_{4}, E\right)$ is called soft $\mathrm{q} T_{1}$ space.

Definition 5.4. Let $\left(X, \tau_{1}, \tau_{2}, \tau_{3}, \tau_{4}, E\right)$ be a soft quad topological space over $\mathrm{X}$ and $x, y \in X$ such that $x \neq y$. If we can find two q-open soft sets such that $x \in(F, E)$ and $y \in(G, E)$ moreover $(F, E) \cap(G, E)=\phi$. Then $\left(X, \tau_{1}, \tau_{2}, \tau_{3}, \tau_{4}, E\right)$ is called a soft $q T_{2}$ space.

Definition 5.5. Let $\left(X, \tau_{1}, \tau_{2}, \tau_{3}, \tau_{4}, E\right)$ be a soft topological space $(G, E)$ be q-closed soft set in $X$ and $x \in X_{A}$ such that $x \notin(G, E)$. If there occurs soft q-open sets $\left(F_{1}, E\right)$ and $\left(F_{2}, E\right)$ such that $x \in\left(F_{1}, E\right),(G, E) \subseteq\left(F_{2}, E\right)$ and $\left(F_{1}, E\right) \cap\left(F_{2}, E\right)=\varphi$. Then $\left(X, \tau_{1}, \tau_{2}, \tau_{3}, \tau_{4}, E\right)$ is called soft q-regular spaces. A soft q-regular $q T_{1}$ Space is called soft $q T_{3}$ space.

Then $\left(X, \tau_{1}, \tau_{2}, \tau_{3}, \tau_{4}, E\right)$ is called a soft q- regular spaces. A soft q-regular $T_{1}$ Space.is called soft $q T_{3}$ space.

Definition 5.6. $\left(X, \tau_{1}, \tau_{2}, \tau_{3}, \tau_{4}, E\right)$ be a soft quad topological space $\left(F_{1}, E\right),(G, E)$ be closed soft sets in $X$ such that

$(F, E) \cap(G, E)=\varphi$ If there exists q-open soft sets $\left(F_{1}, E\right)$ and $\left(F_{2}, E\right)$ such that $(F, E) \subseteq\left(F_{1}, E\right),(G, E) \subseteq\left(F_{2}, E\right)$ and $\left(F_{1}, E\right) \cap\left(F_{2}, E\right)=\varphi$. Then $\left(X, \tau_{1}, \tau_{2}, \tau_{3}, \tau_{4}, E\right)$ is called a q-soft normal space. A soft q-normal $q T_{1}$ Space is called soft $q T_{4}$ Space.

Definition 5.7. Let $(X, \tau, A)$ be a soft Topological space over $\mathrm{X}$ and $e_{G}, e_{H} \in$ $X_{A}$ such that $e_{G} \neq e_{H}$ if there can happen at least one soft semi open set $\left(F_{1}, A\right)$ or $\left(F_{2}, A\right)$ such that $e_{G} \in\left(F_{1}, A\right), e_{H} \notin\left(F_{1}, A\right)$ or $e_{H} \in\left(F_{2}, A\right), e_{G} \notin$ $\left(\left(F_{2}, A\right)\right.$ then $\left(X, \tau_{1}, \tau_{2}, \tau_{3}, \tau_{4}, E\right)$ is called a soft $q T_{0}$ space.

Definition 5.8. Let $\left(X, \tau_{1}, \tau_{2}, \tau_{3}, \tau_{4}, E\right)$ be a soft Topological spaces over $X$ and $e_{G}, e_{H} \in X_{A}$ such that $e_{G} \neq e_{H}$ if there can happen soft q-open sets $\left(F_{1}, A\right)$ and $\left(F_{2}, A\right)$ such that $e_{G} \in\left(F_{1}, A\right), e_{H} \notin\left(F_{1}, A\right)$ and $e_{H} \in\left(F_{2}, A\right)$, $e_{G} \notin\left(\left(F_{2}, A\right)\right.$ then $\left(X, \tau_{1}, \tau_{2}, \tau_{3}, \tau_{4}, E\right)$ is called soft $q T_{1}$ space.

Definition 5.9. Let $\left(X, \tau_{1}, \tau_{2}, \tau_{3}, \tau_{4}, E\right)$ be a soft Topological space over $X$ and $e_{G}, e_{H} \in X_{A}$ such that $e_{G} \neq e_{H}$ if there can happen soft q-open sets $\left(F_{1}, A\right)$ and $\left(F_{2}, A\right)$ such that $e_{G} \in\left(F_{1}, A\right)$, and $e_{H} \in\left(F_{2}, A\right)$

$\left(F_{1}, A\right) \cap\left(F_{2}, A\right)=\phi_{A}$. Then $\left(X, \tau_{1}, \tau_{2}, \tau_{3}, \tau_{4}, E\right)$ is called soft $q T_{2}$ space

Definition 5.10. Let $\left(X, \tau_{1}, \tau_{2}, \tau_{3}, \tau_{4}, E\right)$ be a soft topological space $(G, E)$ be q-closed soft set in $\mathrm{X}$ and $e_{G} \in X_{A}$ such that $e_{G} \notin(G, E)$. If there occurs soft q-open sets $\left(F_{1}, E\right)$ and $\left(F_{2}, E\right)$ such that $e_{G} \in\left(F_{1}, E\right),(G, E) \subseteq\left(F_{2}, E\right)$ and $\left(F_{1}, E\right) \cap\left(F_{2}, E\right)=\varphi$. Then $\left(X, \tau_{1}, \tau_{2}, \tau_{3}, \tau_{4}, E\right)$ is called soft q-regular spaces. A soft q-regular $q T_{1}$ Space is called soft $q T_{3}$ space.

\section{SOF AXIOMS WITH RESPECT TO CRISP POINTS}

In this section separation axioms in quad soft topological structures are examined with respect to ordinary points and different related results are also examined [24].

Definition 6.1. In a soft quad topological space $\left(X, \tau_{1}, \tau_{2}, \tau_{3}, \tau_{4}, E\right)$ 1) $\tau_{1} \cup \tau_{2}$ is said to be soft $T_{0}$ space with respect to $\tau_{3} \cup \tau_{4}$ if for each pair of points $x, y \in X$ such that $x \neq y$ there exists $\tau_{1} \cup \tau_{2}$ soft open $\operatorname{set}(F, E)$ and a $\tau_{3} \cup \tau_{4}$ soft open set $(G, E)$ such that $x \in(F, E)$ and $y \notin(G, E)$ or $y \in(G, E)$ and $x \notin(G, E)$. Similarly, $\tau_{3} \cup \tau_{4}$ is said to be soft $T_{0}$ space with respect to $\tau_{1} \cup \tau_{2}$ if for each pair of points $x, y \in X$ such that $x \neq y$ there exists $\tau_{3} \cup \tau_{4}$ soft open set $(F, E)$ and $\tau_{1} \cup \tau_{2}$ soft open set $(G, E)$ such that $x \in(F, E)$ and $y \notin(F, E)$ or $y \in(G, E)$ and $x \notin(G, E)$. Soft quad topological spaces $\left(X, \tau_{1}, \tau_{2}, \tau_{3}, \tau_{4}, E\right)$ is said to be pair wise soft $T_{0}$ space if $\tau_{1} \cup \tau_{2}$ is soft $T_{0}$ space with respect to $\tau_{3} \cup \tau_{4}$ and to $\tau_{3} \cup \tau_{4}$ and is soft $T_{0}$ space with respect to $\tau_{1} \cup \tau_{2}$.

2) $\tau_{1} \cup \tau_{2}$ is said to be soft $T_{1}$ space with respect to $\tau_{3} \cup \tau_{4}$ if for each pair of points $x, y \in X$ such that $x \neq y$ there exists $\tau_{1} \cup \tau_{2} \operatorname{soft}$ open $\operatorname{set}(F, E)$ and to $\tau_{3} \cup \tau_{4}$ soft open set $(G, E)$ such that $x \in(F, E)$ and $y \notin(G, E)$ and $y \in(G, E)$ and $x \notin(G, E)$. Similarly, $\tau_{3} \cup \tau_{4}$ is said to be soft $T_{1}$ space with respect to $\tau_{1} \cup \tau_{2}$ if for each pair of distinct points $x, y \in X$ such that $x \neq$ $y$ there exists $\tau_{3} \cup \tau_{4}$ soft open set $(F, E)$ and a to $\tau_{1} \cup \tau_{2} \quad$ soft open set $(G, E)$ such that $x \in(F, E)$ and $y \notin(F, E)$ and $y \in(G, E)$ and $x \notin$ $(G, E)$. Soft quad topological spaces $\left(X, \tau_{1}, \tau_{2}, \tau_{3}, \tau_{4}, E\right)$ is said to be pair wise soft $T_{1}$ space if $\tau_{1} \cup \tau_{2}$ is soft $T_{1}$ space with respect to $\tau_{3} \cup \tau_{4}$ and to $\tau_{3} \cup \tau_{4}$ is soft $T_{1}$ space with respect to $\tau_{1} \cup \tau_{2}$. 
3) $\tau_{1} \cup \tau_{2}$ is said to be soft $T_{2}$ space with respect to $\tau_{1} \cup \tau_{2}$ if for each pair of points $x, y \in X$ such that $x \neq y$ there exists a $\tau_{1} \cup \tau_{2}$ soft open set $(F, E)$ and a $\tau_{3} \cup \tau_{4}$ soft open set $(G, E)$ such that $x \in(F, E)$ and $y \in(G, E)$ , $(F, E) \cap(G, E)=\phi$. Similarly, $\tau_{3} \cup \tau_{4}$ is said to be soft $T_{2}$ space with respect to $\tau_{1} \cup \tau_{2}$ if for each pair of points $x, y \in X$ such that $x \neq y$ there exists a $\tau_{3} \cup \tau_{4}$ soft open set $(F, E)$ and a $\tau_{1} \cup \tau_{2}$ soft open set $(G, E)$ such that $x \in(F, E), y \in(G, E)$ and $(F, E) \cap(G, E)=\phi$. The soft quad topological space $\left(X, \tau_{1}, \tau_{2}, \tau_{3}, \tau_{4}, E\right)$ is said to be pair wise soft $T_{2}$ space if $\tau_{1} \cup \tau_{2}$ is soft $T_{2}$ space with respect to $\tau_{3} \cup \tau_{4}$ and $\tau_{3} \cup \tau_{4}$ is soft $T_{2}$ space with respect to $\tau_{1} \cup \tau_{2}$

Definition 6.2. In a soft quad topological space $\left(X, \tau_{1}, \tau_{2}, \tau_{3}, \tau_{4}, E\right)$

1) $\tau_{1} \cup \tau_{2}$ is said to be soft $T_{3}$ space with respect to a $\tau_{3} \cup \tau_{4}$ if $\tau_{1} \cup \tau_{2}$ is soft $T_{1}$ space with respect to $\tau_{3} \cup \tau_{4}$ and for each pair of points $x, y \in X$ such that $x \neq y$ there exists $\tau_{1} \cup \tau_{2}$

Soft closed set $(G, E)$ such that $x \notin(G, E)$, a $\tau_{1} \cup \tau_{2}$ soft open set $\left(F_{1}, E\right)$ and $\tau_{3} \cup \tau_{4}$ soft open set $\left(F_{2}, E\right)$ such that $x \in\left(F_{1}, E\right),(G, E) \subseteq\left(F_{2}, E\right)$ and $\left(F_{1}, E\right) \cap\left(F_{2}, E\right)=\emptyset$. Similarly, $\tau_{3} \cup \tau_{4}$ is said to be soft $T_{3}$ space with respect to $\tau_{1} \cup \tau_{2}$ if $\tau_{3} \cup \tau_{4}$ is soft $T_{1}$ space with respect to $\tau_{1} \cup \tau_{2}$ and for each pair of points $x, y \in X$ such that $x \neq y$ there exists a $\tau_{3} \cup \tau_{4}$ soft closed set $(G, E)$ such that $x \notin(G, E), \tau_{3} \cup \tau_{4}$ soft open set $\left(F_{1}, E\right)$ and $\tau_{1} \cup$ $\tau_{2}$ soft open set $\left(F_{2}, E\right)$ such that $x \in\left(F_{1}, E\right),(G, E) \subseteq\left(F_{2}, E\right)$ and $\left(F_{1}, E\right) \cap$ $\left(F_{2}, E\right)=\phi .\left(X, \tau_{1}, \tau_{2}, \tau_{3}, \tau_{4}, E\right)$ is said to be pair wise soft $T_{3}$ space if $\tau_{1} \cup$ $\tau_{2}$ is soft $T_{3}$ space with respect to $\tau_{3} \cup \tau_{4}$ and $\tau_{3} \cup \tau_{4}$ is soft $T_{3}$ space with respect to $\tau_{1} \cup \tau_{2}$

2) $\tau_{1} \cup \tau_{2}$ is said to be soft $T_{4}$ space with respect to $\tau_{3} \cup \tau_{4}$ if $\tau_{1} \cup \tau_{2}$ is soft $T_{1}$ space with respect to $\tau_{3} \cup \tau_{4}$, there exists a $\tau_{1} \cup \tau_{2}$ soft closed set $\left(F_{1}, E\right)$ and $\tau_{3} \cup \tau_{4}$ soft closed set $\left(F_{2}, E\right)$ such that $\left(F_{1}, E\right) \cap\left(F_{2}, E\right)=$ $\emptyset$. Also there exists $\left(F_{3}, E\right)$ and $\left(G_{1}, E\right)$ such that $\left(F_{3}, E\right)$ is soft $\tau_{1} \cup \tau_{2}$ open set, $\left(G_{1}, E\right)$ is soft $\tau_{3} \cup \tau_{4}$ open set such that $\left(F_{1}, E\right) \subseteq\left(F_{3}, E\right),\left(F_{2}, E\right) \subseteq$ $\left(G_{1}, E\right)$. Similarly, $\tau_{3} \cup \tau_{4}$ is said to be soft $T_{4}$ space with respect to $\tau_{1} \cup \tau_{2}$ if $\tau_{3} \cup \tau_{4}$ is soft $T_{1}$ space with respect to $\tau_{1} \cup \tau_{2}$, there exists $\tau_{3} \cup \tau_{4}$ soft closed set $\left(F_{1}, E\right)$ and $\tau_{1} \cup \tau_{2}$ soft closed set $\left(F_{2}, E\right)$ such that $\left(F_{1}, E\right) \cap$ $\left(F_{2}, E\right)=\phi$. Also there exist $\left(F_{3}, E\right)$ and $\left(G_{1}, E\right)$ such that $\left(F_{3}, E\right)$ is soft $\tau_{3} \cup \tau_{4}$ open set, $\left(G_{1}, E\right)$ is soft $\tau_{1} \cup \tau_{2}$ open set such that $\left(F_{1}, E\right) \subseteq$ $\left(F_{3}, E\right),\left(F_{2}, E\right) \subseteq\left(G_{1}, E\right)$ and $\left(F_{3}, E\right) \cap\left(G_{1}, E\right)=\phi$. Thus, $\left(X, \tau_{1}, \tau_{2}, E\right)$ is said to be pair wise soft $T_{4}$ space if $\tau_{1} \cup \tau_{2}$ is soft $T_{4}$ space with respect to $\tau_{3} \cup \tau_{4}$ and $\tau_{3} \cup \tau_{4}$ is soft $T_{4}$ space with respect to $\tau_{1} \cup \tau_{2}$.

Proposition 9. Let $\left(X, \tau_{1}, \tau_{2}, \tau_{3}, \tau_{4}, E\right)$ be a soft quad topological space over $X$. Then, if $\left(X, \tau_{1}, \tau_{2}, E\right)$ and $\left(X, \tau_{3}, \tau_{4}, E\right)$ are soft $T_{3}$ space then $\left(X, \tau_{1}, \tau_{2}, \tau_{3}, \tau_{4}, E\right)$ is a pair wise soft $T_{2}$ space.

Proof: Suppose $\left(X, \tau_{1}, \tau_{2}, E\right)$ is a soft $T_{3}$ space with respect to $\left(X, \tau_{3}, \tau_{4}, E\right)$ then according to definition for $x, y \in X$, which distinct, by using Proposition $1,(\mathrm{Y}, \mathrm{E})$ is soft closed set in $\tau_{3} \cup \tau_{4}$ and $x \notin(Y, E)$ there exists a $\tau_{1} \cup \tau_{2}$ soft $\beta$ open set $(F, E)$ and a $\tau_{3} \cup \tau_{4}$ soft open set $(G, E)$ such that $x \in(F, E), y \in(Y, E) \subseteq(G, E)$ and $\left(F_{1}, E\right) \cap\left(F_{2}, E\right)=\phi$. Hence $\tau_{1} \cup \tau_{2}$ is soft $T_{2}$ space with respect to $\tau_{3} \cup \tau_{4}$. Similarly, if $\left(X, \tau_{3}, \tau_{4}, E\right)$ is a soft $T_{3}$ space with respect to $\left(X, \tau_{1}, \tau_{2}, E\right)$ then according to definition for $x, y \in$ $X, x \neq y$, by using Theorem $2,(\mathrm{x}, \mathrm{E})$ is closed soft set in $\tau_{1} \cup \tau_{2}$ and $y \notin$ $(x, E)$ there exists a $\tau_{3} \cup \tau_{4}$ soft open set $(F, E)$ and a $\tau_{1} \cup \tau_{2}$ soft open set $(G, E)$ such that $y \in(F, E), x \in(x, E) \subseteq(G, E)$ and $\left(F_{1}, E\right) \cap\left(F_{2}, E\right)=\phi$ .Hence $\tau_{3} \cup \tau_{4}$ is soft $T_{2}$ space. This implies that $\left(X, \tau_{1}, \tau_{2}, \tau_{3}, \tau_{4}, E\right)$ is a pair wise soft $T_{2}$ space.

Proposition 10. Let $\left(X, \tau_{1}, \tau_{2}, \tau_{3}, \tau_{4}, E\right)$ be a soft quad topological space over $X$. if $\left(X, \tau_{1}, \tau_{2}, E\right)$ and $\left(X, \tau_{3}, \tau_{4}, E\right)$ are soft $T_{3}$ space then $\left(X, \tau_{1}, \tau_{2}, \tau_{3}, \tau_{4}, E\right)$ is a pair wise soft $T_{3}$ space.

Proof: Suppose $\left(X, \tau_{1}, \tau_{2}, E\right)$ is a soft $T_{3}$ space with respect to $\left(X, \tau_{3}, \tau_{4}, E\right)$ then according to definition for $x, y \in X, x \neq y$ there exists $\mathrm{a}\left(X, \tau_{1}, \tau_{2}, E\right)$ soft open set $(F, E)$ and a $\left(X, \tau_{3}, \tau_{4}, E\right)$ soft open set $(G, E)$ such that $x \in$ $(F, E)$ and $y \notin(F, E)$ or $y \in(G, E)$ and $x \notin(G, E)$ and for each point $x \in$ $X$ and each $\left(X, \tau_{1}, \tau_{2}, E\right)$ closed soft set $\left(G_{1}, E\right)$ such that $x \notin\left(G_{1}, E\right)$ there exists a $\left(X, \tau_{1}, \tau_{2}, E\right)$ soft open set $\left(F_{1}, E\right)$ and $\left(X, \tau_{3}, \tau_{4}, E\right)$ soft open set $\left(F_{2}, E\right)$ such that $x \in\left(F_{1}, E\right),\left(G_{1}, E\right) \subseteq\left(F_{2}, E\right)$ and $\left(F_{1}, E\right) \cap\left(F_{2}, E\right)=\phi$. Similarly, to $\left(X, \tau_{3}, \tau_{4}, E\right)$ is a soft $T_{3}$ space with respect to $\left(X, \tau_{1}, \tau_{2}, E\right)$ So according to definition for $x, y \in X, x \neq y$ there exists a $\left(X, \tau_{3}, \tau_{4}, E\right)$ soft open set $(F, E)$ and a $\left(X, \tau_{1}, \tau_{2}, E\right)$ soft open set $(G, E)$ such that $x \in(F, E)$ and $y \notin(F, E)$ or $y \in(G, E)$ and $x \notin(G, E)$ and for each point $x \in X$ and each $\left(X, \tau_{3}, \tau_{4}, E\right)$ closed soft set $\left(G_{1}, E\right)$ such that $x \notin\left(G_{1}, E\right)$ there exists $\left(X, \tau_{3}, \tau_{4}, E\right)$ soft $\beta$ open set $\left(F_{1}, E\right)$ and $\left(X, \tau_{1}, \tau_{2}, E\right)$ soft open set $\left(F_{2}, E\right)$ such that $x \in\left(F_{1}, E\right),\left(G_{1}, E\right) \subseteq\left(F_{2}, E\right)$ and $\left(F_{1}, E\right) \cap\left(F_{2}, E\right)=\phi$. Hence $\left(X, \tau_{1}, \tau_{2}, \tau_{3}, \tau_{4}, E\right)$ is pair wise soft $T_{3}$ space.

Proposition 11. If $\left(X, \tau_{1}, \tau_{2}, \tau_{3}, \tau_{4}, E\right)$ be a soft quad topological space over $\mathrm{X}$. if $\left(X, \tau_{1}, \tau_{2}, E\right)$ and $\left(X, \tau_{3}, \tau_{4}, E\right)$ are soft $T_{4}$ space then $\left(X, \tau_{1}, \tau_{2}, \tau_{3}, \tau_{4}, E\right)$ is pair wise soft $T_{4}$ space.

Proof: Suppose $\left(X, \tau_{1}, \tau_{2}, E\right)$ is soft $T_{4}$ space with respect to
$\left(X, \tau_{3}, \tau_{4}, E\right)$.So according to definition for $x, y \in X, x \neq y$ there exist a $\left(X, \tau_{1}, \tau_{2}, E\right)$ soft open set $(F, E)$ and $\mathrm{a}\left(X, \tau_{3}, \tau_{4}, E\right)$ soft open set $(G, E)$ such that $x \in(F, E)$ and $y \notin(F, E)$ or $y \in(G, E)$ and $x \notin(G, E)$ each $\left(X, \tau_{1}, \tau_{2}, E\right)$ soft closed set $\left(F_{1}, E\right)$ and a $\left(X, \tau_{3}, \tau_{4}, E\right)$ soft closed set $\left(F_{2}, E\right)$ such that $\left(F_{1}, E\right) \cap\left(F_{2}, E\right)=\phi$. There exist $\left(F_{3}, E\right)$ and $\left(G_{1}, E\right)$ such that $\left(F_{3}, E\right)$ is soft $\left(X, \tau_{3}, \tau_{4}, E\right)$ and soft open set $\left(G_{1}, E\right)$ is soft $\left(X, \tau_{1}, \tau_{2}, E\right)$ open set $\left(F_{1}, E\right) \subseteq\left(F_{3}, E\right),\left(F_{2}, E\right) \subseteq\left(G_{1}, E\right)$ and $\left(F_{3}, E\right) \cap\left(G_{1}, E\right)=\phi$. Similarly, $\left(X, \tau_{3}, \tau_{4}, E\right)$ is soft $T_{4}$ space with respect to $\left(X, \tau_{1}, \tau_{2}, E\right)$ is so according to definition for $x, y \in X, x \neq y$ there exists a $\left(X, \tau_{3}, \tau_{4}, E\right)$ soft semi open set $(F, E)$ and a $\left(X, \tau_{1}, \tau_{2}, E\right)$ soft open set $(G, E)$ such that $x \in$ $(F, E)$ and $y \notin(F, E)$ or $y \in(G, E)$ and $x \notin(G, E)$ and for each $\left(X, \tau_{3}, \tau_{4}, E\right)$ soft closed set $\left(F_{1}, E\right)$ and $\left(X, \tau_{1}, \tau_{2}, E\right)$ soft closed set $\left(F_{2}, E\right)$ such that $\left(F_{1}, E\right) \cap\left(F_{2}, E\right)=\phi$.there exists soft open sets $\left(F_{3}, E\right)$ and $\left(G_{1}, E\right)$ such that $\left(F_{3}, E\right)$ is soft $\left(X, \tau_{3}, \tau_{4}, E\right) \beta$ open set $\left(G_{1}, E\right)$ is soft $\left(X, \tau_{1}, \tau_{2}, E\right)$ open set such that $\left(F_{1}, E\right) \subseteq\left(F_{3}, E\right),\left(F_{2}, E\right) \subseteq\left(G_{1}, E\right)$ and $\left(F_{3}, E\right) \cap\left(G_{1}, E\right)=\phi$. Hence $\left(X, \tau_{1}, \tau_{2}, \tau_{3}, \tau_{4}, E\right)$ is pair wise soft $T_{4}$ space.

Proposition 12. Let $\left(X, \tau_{1}, \tau_{2}, \tau_{3}, \tau_{4}, E\right)$ be a soft quad topological space over $\mathrm{X}$ and $\mathrm{Y}$ be a non-empty subset of $\mathrm{X}$. if $\left(X, \tau_{1 Y}, \tau_{2 Y}, \tau_{3 Y}, \tau_{4 Y}, E\right)$ is pair wise soft $T_{3}$ space. Then $\left(Y, \tau_{1 Y}, \tau_{2 Y}, \tau_{3 Y}, \tau_{4 Y}, E\right)$ is pair wise soft $T_{3}$ space.

Proof: First we prove that $\left(X, \tau_{1 Y}, \tau_{2 Y}, \tau_{3 Y}, \tau_{4 Y}, E\right)$ is pair wise soft $T_{1}$ space. Let $x, y \in X, x \neq y$ if $\left(X, \tau_{1}, \tau_{2}, \tau_{3}, \tau_{4}, E\right)$ is pair wise space then this implies that $\left(X, \tau_{1}, \tau_{2}, \tau_{3}, \tau_{4}, E\right)$ is pair wise soft space. So there exists $\left(X, \tau_{1}, \tau_{2}, E\right)$ soft open $(F, E)$ and $\left(X, \tau_{3}, \tau_{4}, E\right)$ soft open set $(G, E)$ such that $x \in(F, E)$ and $y \notin(F, E)$ or $y \in(G, E)$ and $x \notin(G, E)$ now $x \in$ $Y$ and $x \notin(G, E)$. Hence $x \in Y \cap(F, E)=\left(Y_{F}, E\right)$ then $y \notin Y \cap(\alpha)$ for some $\alpha \in E$. this means that $\alpha \in E$ then $y \notin Y \cap F(\alpha)$ for some $\alpha \in E$. Therefore, $y \notin Y \cap(F, E)=\left(Y_{F}, E\right)$. Now $y \in Y$ and $y \in(G, E)$. Hence $y \in$ $Y \cap(G, E)=\left(G_{Y}, E\right)$ where $(G, E) \in\left(X, \tau_{3}, \tau_{4}, E\right)$. Consider $x \notin(G, E)$ this means that $\alpha \in E$ then $x \notin Y \cap G(\alpha)$ for some $\alpha \in E$. There fore $x \notin Y \cap$ $(G, E)=\left(G_{Y}, E\right)$ thus $\left(Y, \tau_{1 Y}, \tau_{2 Y}, \tau_{3 Y}, \tau_{4 Y}, E\right)$ is pair wise soft $T_{1}$ space. Now we prove that $\left(Y, \tau_{1 Y}, \tau_{2 Y}, \tau_{3 Y}, \tau_{4 Y}, E\right)$ is pair wise soft $T_{3}$ space then $\left(Y, \tau_{1 Y}, \tau_{2 Y}, \tau_{3 Y}, \tau_{4 Y}, E\right)$ is pair wise soft regular space.

Let $y \in Y$ and $(G, E)$ be a soft closed set in $Y$ such that $y \notin(G, E)$ where $(G, E) \in\left(X, \tau_{1}, \tau_{2}, \tau_{3}, \tau_{4}, E\right)$ then $(G, E)=(Y, E) \cap(F, E)$ for some soft closed set $\operatorname{in}\left(X, \tau_{1 Y}, \tau_{2 Y}, \tau_{3 Y}, \tau_{4 Y}, E\right)$. Hence $y \notin(Y, E) \cap(F, E)$ but $y \in$ $(Y, E)$, so $y \notin(F, E)$ since $\left(X, \tau_{1}, \tau_{2}, \tau_{3}, \tau_{4}, E\right)$ is soft $T_{3}$ space

$\left(X, \tau_{1}, \tau_{2}, \tau_{3}, \tau_{4}, E\right)$ is soft regular space so there exists $\left(X, \tau_{1}, \tau_{2}, E\right)$ soft open set $\left(F_{1}, E\right)$ and $\left(X, \tau_{3}, \tau_{4}, E\right)$ soft open set $\left(F_{2}, E\right)$ such that

$$
y \in\left(F_{1}, E\right),(G, E) \subseteq\left(F_{2}, E\right)
$$$$
\left(F_{1}, E\right)\left(F_{2}, E\right)=\phi
$$

Take $\left(G_{1}, E\right)=(Y, E) \cap\left(F_{2}, E\right)$ then $\left(G_{1}, E\right),\left(G_{2}, E\right)$ are soft open set in $\mathrm{Y}$ such that

$$
\begin{aligned}
& y \in\left(G_{1}, E\right),(G, E) \subseteq(Y, E) \cap\left(F_{2}, E\right) \\
&=\left(G_{2}, E\right) \\
&\left(G_{1}, E\right) \cap\left(G_{2}, E\right) \subseteq\left(F_{1}, E\right) \cap\left(F_{2}, E\right)=\phi \\
&\left(G_{1}, E\right) \cap\left(G_{2}, E\right)=\phi
\end{aligned}
$$

There fore $\tau_{1 Y} \cup \tau_{2 Y}$ is soft regular space with respect to $\tau_{3 Y} \cup \tau_{4 Y}$. Similarly, Let $y \in Y$ and $(G, E)$ be a soft closed sub set in Y such that $y \notin(G, E)$, where $(G, E) \in\left(X, \tau_{3}, \tau_{4}, E\right)$ then $(G, E)=(Y, E) \cap(F, E)$ where $(F, E)$ is some soft closed set $\operatorname{in}\left(X, \tau_{3}, \tau_{4}, E\right) . \quad y \notin(Y, E) \cap(F, E)$ But $y \in(Y, E)$ so $y \notin$ $(F, E)$ since $\left(X, \tau_{1}, \tau_{2}, E\right)$ is soft regular space so there exists $\left(X, \tau_{3}, \tau_{4}, E\right)$ soft $\beta$ open set $\left(F_{1}, E\right)$ and $\left(X, \tau_{1}, \tau_{2}, E\right)$ soft open $\operatorname{set}\left(F_{2}, E\right)$. Such that

$$
y \in\left(F_{1}, E\right),(G, E) \subseteq\left(F_{2}, E\right)
$$

Take

$$
\left(F_{1}, E\right) \cap\left(F_{2}, E\right)=\phi
$$

$$
\left(G_{1}, E\right)=(Y, E) \cap\left(F_{1}, E\right)
$$$$
\left(G_{1}, E\right)=(Y, E) \cap\left(F_{1}, E\right)
$$

Then $\left(G_{1}, E\right)$ and $\left(G_{2}, E\right)$ are soft open set in $Y$ such that

$$
y \in\left(G_{1}, E\right),(G, E) \subseteq(Y, E) \cap\left(F_{2}, E\right)=
$$

$$
\left(G_{1}, E\right) \cap\left(G_{2}, E\right) \subseteq\left(F_{1}, E\right) \cap\left(F_{2}, E\right)=\phi
$$

There fore $\tau_{3 Y} \cup \tau_{4 Y}$ is soft regular space with respect $\tau_{1 Y} \cup \tau_{2 Y}$ $\Rightarrow\left(Y, \tau_{1 Y}, \tau_{2 Y}, \tau_{3 Y}, \tau_{4 Y}, E\right)$ is pair wise soft $T_{3}$ space.

Proposition 13. Let $\left(X, \tau_{1}, \tau_{2}, \tau_{3}, \tau_{4}, E\right)$ be a soft quad topological space over $X$ and $Y$ be a soft closed sub space of $X$. if $\left(X, \tau_{1}, \tau_{2}, \tau_{3}, \tau_{4}, E\right)$ is pair wise soft $T_{4}$ space then $\left(Y, \tau_{1 Y}, \tau_{2 Y}, \tau_{3 Y}, \tau_{4 Y}, E\right)$ is pair wise soft $T_{4}$ space.

Proof: Since $\left(X, \tau_{1}, \tau_{2}, \tau_{3}, \tau_{4}, E\right)$ is pair wise soft $T_{4}$ space so this implies that $\left(X, \tau_{1}, \tau_{2}, \tau_{3}, \tau_{4}, E\right)$ is pair wise soft $T_{1}$ space as proved above. We prove $\left(X, \tau_{1}, \tau_{2}, \tau_{3}, \tau_{4}, E\right)$ is pair wise soft normal space. Let $\left(G_{1}, E\right),\left(G_{2}, E\right)$ be soft closed sets in Y such that

$\begin{array}{ll} & \left(G_{1}, E\right) \cap\left(G_{2}, E\right)=\phi \\ \text { Then } & \left(G_{1}, E\right)=(Y, E) \cap\left(F_{1}, E\right) \\ \text { And } & \left(G_{2}, E\right)=(Y, E) \cap\left(F_{2}, E\right)\end{array}$

For some soft closed sets such that $\left(F_{1}, E\right)$ is soft closed set in $\tau_{1} \cup \tau_{2}$ soft closed set $\left(F_{2}, E\right)$ in $\tau_{3} \cup \tau_{4}$

And

$\left(F_{1}, E\right) \cap\left(F_{2}, E\right)=\phi$

From Proposition 2. Since, $\mathrm{Y}$ is soft closed sub set of $\mathrm{X}$ then $\left(G_{1}, E\right),\left(G_{2}, E\right)$ are soft closed sets in $\mathrm{X}$ such that 


\section{$\left(G_{1}, E\right) \cap\left(G_{2}, E\right)=\phi$}

Since $\left(X, \tau_{1}, \tau_{2}, \tau_{3}, \tau_{4}, E\right)$ is pair wise soft $\beta$ normal space. So there exists soft open sets $\left(H_{1}, E\right)$ and $\left(H_{2}, E\right)$ such that

$\left(H_{1}, E\right)$ is soft open set in $\tau_{1} \cup \tau_{2}$ and $\left(H_{2}, E\right)$ is soft open set in $\tau_{3} \cup \tau_{4}$ such that

$\left(G_{1}, E\right) \subseteq\left(H_{1}, E\right)$

$\left(G_{2}, E\right) \subseteq\left(H_{2}, E\right)$

$\left(H_{1}, E\right) \cap\left(H_{2}, E\right)=\phi$

Since $\quad\left(G_{1}, E\right),\left(G_{2}, E\right) \subseteq(Y, E)$

Then

$\left(G_{1}, E\right) \subseteq(Y, E) \cap\left(H_{1}, E\right)$

$\left(G_{2}, E\right) \subseteq(Y, E) \cap\left(H_{2}, E\right)$

And $\quad\left[(Y, E) \cap\left(H_{1}, E\right)\right] \cap\left[(Y, E) \cap\left(H_{2}, E\right)\right]=\phi$
Where $(Y, E) \cap\left(H_{1}, E\right)$ and $(Y, E) \cap\left(H_{2}, E\right)$ are soft open sets in $Y$ there fore

$\tau_{1 Y} \cup \tau_{2 Y}$ is soft normal space with respect to $\tau_{3 Y} \cup \tau_{4 Y}$. Similarly, let $\left(G_{1}, E\right),\left(G_{2}, E\right)$ be soft closed sub set in $Y$ such that

$\begin{array}{ll}\text { Then } & \left(G_{1}, E\right) \cap\left(G_{2}, E\right)=\phi \\ & \left(G_{1}, E\right)=(Y, E) \cap\left(F_{1}, E\right)\end{array}$

And $\quad\left(G_{2}, E\right)=(Y, E) \cap\left(F_{2}, E\right)$

For some soft closed sets such that $\left(F_{1}, E\right)$ is soft closed set in $\tau_{3} U$ $\tau_{4}$ and $\left(F_{2}, E\right)$ soft closed set in $\tau_{1} \cup \tau_{2}$ and

$$
\left(F_{1}, E\right)\left(F_{2}, E\right)=\phi
$$

From Proposition 2. Since, $\mathrm{Y}$ is soft closed sub set in $\mathrm{X}$ then $\left(G_{1}, E\right),\left(G_{2}, E\right)$ are soft closed sets in $\mathrm{X}$ such that

$$
\left(G_{1}, E\right) \cap\left(G_{2}, E\right)=\phi
$$

Since $\left(X, \tau_{1}, \tau_{2}, \tau_{1}, \tau_{2}, E\right)$ is pair wise soft normal space so there exists soft open sets $\left(H_{1}, E\right)$ and $\left(H_{2}, E\right)$

Such that $\left(H_{1}, E\right)$ is soft open set is $\tau_{3} \cup \tau_{4}$ and $\left(H_{2}, E\right)$ is soft open set in $\tau_{1} \cup$ $\tau_{2}$ such that

$\begin{array}{ll} & \left(G_{1}, E\right) \subseteq\left(H_{1}, E\right) \\ & \left(G_{2}, E\right) \subseteq\left(H_{2}, E\right) \\ & \left(H_{1}, E\right) \cap\left(H_{2}, E\right)=\phi \\ & \left(G_{1}, E\right),\left(G_{2}, E\right) \subseteq(Y, E) \\ \text { Since } & \left(G_{1}, E\right) \subseteq(Y, E) \cap\left(H_{1}, E\right) \\ \text { Then } & \left(G_{2}, E\right) \subseteq(Y, E) \cap\left(H_{2}, E\right) \\ & {\left[(Y, E) \cap\left(H_{1}, E\right)\right] \cap\left[(Y, E) \cap\left(H_{2}, E\right)\right]=\phi}\end{array}$

Where $(Y, E) \cap\left(H_{1}, E\right)$ and $(Y, E) \cap\left(H_{2}, E\right)$ are soft $\beta$ open sets in $Y$ there fore $\tau_{3 Y} \cup \tau_{4 Y}$ is soft normal space with respect to $\tau_{1 Y} \cup \tau_{2 Y}$

$\Rightarrow\left(Y, \tau_{1 Y}, \tau_{2 Y}, \tau_{3 Y}, \tau_{4 Y} E\right)$ is pair wise soft $T_{4}$ space.

\section{SOFT AXIOMS WITH RESPECT TO SOFT POINTS}

In this section, we brought out soft topological structures known as separation axioms in quad soft topology with respect to soft points. With the applications of these soft separation axioms different result are discussed

Definition 7.1. In a soft quad topological space $\left(X, \tau_{1}, \tau_{2}, \tau_{1}, \tau_{2}, E\right)$

1) $\tau_{1} \cup \tau_{2}$ said to be soft $T_{0}$ space with respect to $\tau_{3} \cup \tau_{4}$ if for each pair of distinct points $e_{G}, e_{H} \in X_{A}$ there happens $\tau_{1} \cup \tau_{2}$ soft open set $(F, E)$ and a $\tau_{3} \cup \tau_{4}$ soft open set $(G, E)$ such that $e_{G} \in(F, E)$ and $e_{H} \notin(G, E)$, Similarly, $\tau_{3} \cup \tau_{4}$ is said to be soft $T_{0}$ space with respect to $\tau_{1} \cup \tau_{2}$ if for each pair of distinct points $e_{G}, e_{H} \in X_{A}$ there happens $\tau_{3} \cup \tau_{4}$ soft open $\operatorname{set}(F, E)$ and a $\tau_{1} \cup \tau_{2}$ soft open set $(G, E)$ such that $e_{G} \in(F, E)$ and $e_{H} \notin$ $(F, E)$ or $e_{H} \in(G, E)$ and $e_{G} \notin(G, E)$. Soft quad topological spaces $\left(X, \tau_{1}, \tau_{2}, \tau_{1}, \tau_{2}, E\right)$ is said to be pair wise soft $T_{0}$ space if $\tau_{1} \cup \tau_{2}$ is soft $T_{0}$ space with respect to $\tau_{3} \cup \tau_{4}$ and $\tau_{3} \cup \tau_{4}$ is soft $T_{0}$ spaces with respect to $\tau_{1} \cup \tau_{2}$.

2) $\tau_{1} \cup \tau_{2}$ is said to be soft $T_{1}$ space with respect to $\tau_{3} \cup \tau_{4}$ if for each pair of distinct points $e_{G}, e_{H} \in X_{A}$ there happens a $\tau_{1} \cup \tau_{2}$ soft open set $(F . E)$ and $\tau_{3} \cup \tau_{4}$ soft open set $(G, E)$ such that $e_{G} \in(F, E)$ and $e_{H} \notin$ $(G, E)$ and $e_{H} \in(G, E)$ and $e_{G} \notin(G, E)$. Similarly, $\tau_{3} \cup \tau_{4}$ is said to be soft $T_{1}$ space with respect to $\tau_{1} \cup \tau_{2}$ if for each pair of distinct points $e_{G}, e_{H} \in$ $X_{A}$ there exist a $\tau_{3} \cup \tau_{4}$ soft open set $(F, E)$ and a $\tau_{1} \cup \tau_{2}$ soft open set $(G, E)$ such that $e_{G} \in(F, E)$ and $e_{H} \notin(G, E)$ and $e_{H} \in(G, E)$ and $e_{G} \notin$ $(G, E)$. Soft quad topological space $\left(X, \tau_{1}, \tau_{2}, \tau_{1}, \tau_{2}, E\right)$ is said to be pair wise soft $T_{1}$ space if $\tau_{1} \cup \tau_{2}$ is soft $T_{1}$ space with respect to $\tau_{3} \cup \tau_{4}$ and $\tau_{3} \cup \tau_{4}$ is soft $T_{1}$ spaces with respect to $\tau_{1} \cup \tau_{2}$.

3) $\tau_{1} \cup \tau_{2}$ is said to be soft $T_{2}$ space with respect to $\tau_{3} \cup \tau_{4}$ if for each pair of distinct points $e_{G}, e_{H} \in X_{A}$ there happens a $\tau_{1} \cup \tau_{2}$ soft open set $(F, E)$ and a $\tau_{3} \cup \tau_{4}$ soft open set $(G, E)$ such that $e_{G} \in(F, E)$ and $e_{H} \notin(G, E)$ and $e_{H} \in(G, E)$ and $e_{G} \notin(G, E)$ and $(F, E) \cap(G, E)=\phi$. Similarly, $\tau_{3} \cup$ $\tau_{4}$ is aid to be soft $T_{2}$ space with respect to $\tau_{1} \cup \tau_{2}$ if for each pair of distinct points $e_{G}, e_{G} \in X_{A}$ there happens a $\tau_{3} \cup \tau_{4}$ soft open set $(F, E)$ and a $\tau_{1} \cup \tau_{2}$ soft open set $(G, E)$ such that $e_{G} \in(F, E)$ and $e_{G} \in(G, E)$ and $(F, E) \cap(G, E)=\phi$. The soft quad topological space $\left(X, \tau_{1}, \tau_{2}, \tau_{1}, \tau_{2}, E\right)$ is said to be pair wise soft $T_{2}$ space if $\tau_{1} \cup \tau_{2}$ is soft $T_{2}$ space with respect to $\tau_{3} \cup \tau_{4}$ and $\tau_{3} \cup \tau_{4}$ is soft $T_{2}$ space with respect to $\tau_{1} \cup \tau_{2}$.

Definition 7.2. In a soft quad topological space $\left(X, \tau_{1}, \tau_{2}, \tau_{1}, \tau_{2}, E\right)$
1) $\tau_{1} \cup \tau_{2}$ is said to be soft $T_{3}$ space with respect to $\tau_{3} \cup \tau_{4}$ if $\tau_{1} \cup \tau_{2}$ is soft $T_{1}$ space with respect to $\tau_{3} \cup \tau_{4}$ and for each pair of distinct points $e_{G}, e_{H} \in X_{A}$, there exists a $\tau_{1} \cup \tau_{2}$ closed soft set $(G, E)$ such that $e_{G} \notin$ $(G, E), \tau_{1} \cup \tau_{2}$ soft open set $\left(F_{1}, E\right)$ and $\tau_{3} \cup \tau_{4}$ soft open set $\left(F_{2}, E\right)$ such that $e_{G} \in\left(F_{1}, E\right),(G, E) \subseteq\left(F_{2}, E\right)$ and $\left(F_{1}, E\right) \cap\left(F_{2}, E\right)=\emptyset$. Similarly, $\tau_{3} \cup$ $\tau_{4}$ is said to be soft $T_{3}$ space with respect to $\tau_{1} \cup \tau_{2}$ if $\tau_{3} \cup \tau_{4}$ is soft $T_{1}$ space with respect to $\tau_{1} \cup \tau_{2}$ and for each pair of distinct points $e_{G}, e_{H} \in$ $X_{A}$ there exists a $\tau_{3} \cup \tau_{4}$ soft open set $(G, E)$ such that $e_{G} \notin(G, E), \tau_{3} \cup \tau_{4}$ soft open set $\left(F_{1}, E\right)$ amd $\tau_{1} \cup \tau_{2}$ soft open set $\left(F_{2}, E\right)$ such that $e_{G} \in$ $\left(F_{1}, E\right),(G, E) \subseteq\left(F_{2}, E\right)$ and $\left(F_{1}, E\right) \cap\left(F_{2}, E\right)=\phi$.

$\left(X, \tau_{1}, \tau_{2}, \tau_{1}, \tau_{2}, E\right)$ is said to be pair wise soft $T_{3}$ space if $\tau_{1} \cup \tau_{2}$ is soft $T_{3}$ space with respect to $\tau_{3} \cup \tau_{4}$ and $\tau_{3} \cup \tau_{4}$ is soft $T_{3}$ space with respect to $\tau_{1} \cup \tau_{2}$.

2) $\tau_{1} \cup \tau_{2}$ is said to be soft $T_{4}$ space with respect to $\tau_{3} \cup \tau_{4}$ if $\tau_{1} \cup \tau_{2}$ is soft $T_{1}$ space with respect to $\tau_{3} \cup \tau_{4}$, there exists a $\tau_{1} \cup \tau_{2}$ soft closed set $\left(F_{1}, E\right)$ and $\tau_{3} \cup \tau_{4}$ soft closed set $\left(F_{2}, E\right)$ such that $\left(F_{1}, E\right) \cap\left(F_{2}, E\right)=$ $\emptyset$,also, there exists $\left(F_{3}, E\right)$ and $\left(G_{1}, E\right)$ such that $\left(F_{3}, E\right)$ is soft $\tau_{1} \cup \tau_{2}$ open set, $\left(G_{1}, E\right)$ is soft $\tau_{3} \cup \tau_{4}$ open set such that $\left(F_{1}, E\right) \subseteq\left(F_{3}, E\right),\left(F_{2}, E\right) \subseteq$ $\left(G_{1}, E\right)$. Similarly, $\tau_{3} \cup \tau_{4}$ is said to be soft $T_{4}$ space with respect to $\tau_{1} \cup \tau_{2}$ if $\tau_{3} \cup \tau_{4}$ is soft $T_{1}$ space with respect to $\tau_{1} \cup \tau_{2}$ there exists $\tau_{3} \cup \tau_{4}$ soft closed set $\left(F_{1}, E\right)$ and $\tau_{1} \cup \tau_{2}$ soft closed set $\left(F_{2}, E\right)$ such that $\left(F_{1}, E\right) \cap$ $\left(F_{2}, E\right)=\phi$. Also there exists $\left(F_{3}, E\right)$ and $\left(G_{1}, E\right)$ such that $\left(F_{3}, E\right)$ is soft $\tau_{3} \cup \tau_{4}$ open set, $\left(G_{1}, E\right)$ is soft $\tau_{1} \cup \tau_{2}$ soft set such that $\left(F_{1}, E\right) \subseteq$ $\left(F_{3}, E\right),\left(F_{2}, E\right) \subseteq\left(G_{1}, E\right) \quad$ and $\quad\left(F_{3}, E\right) \cap\left(G_{1}, E\right)=$ $\phi$. Thus, $\left(X, \tau_{1}, \tau_{2}, \tau_{1}, \tau_{2}, E\right)$ is said to be pair wise soft $T_{4}$ space if $\tau_{1} \cup \tau_{2}$ is soft $T_{4}$ space with respect to $\tau_{3} \cup \tau_{4}$ and $\tau_{3} \cup \tau_{4}$ is soft $T_{4}$ space with respect to $\tau_{1} \cup \tau_{2}$.

Proposition 14. Let $\left(X, \tau_{1}, \tau_{2}, \tau_{3}, \tau_{4}, E\right)$ be a soft topological space over $X .\left(X, \tau_{1}, \tau_{2}, \tau_{3}, \tau_{4}, E\right)$ is soft $T_{3}$ space, then for all $e_{G} \in X_{E} \quad e_{G}=\left(e_{G}, E\right)$ is soft-closed set.

Proof: We want to prove that $e_{G}$ is closed soft set, which is sufficient to prove that $e_{G}^{c}$ is open soft set for all $e_{H} \in\left\{e_{G}\right\}^{c}$.Since $\left(X, \tau_{1}, \tau_{2}, \tau_{3}, \tau_{4}, E\right)$ is soft $T_{3}$ space, then there exists soft sets $(F, E)_{e_{H}}$ and $(G, E)$ such that $e_{H_{E}} \subseteq$ $(F, E)_{e_{H}}$ and $e_{G_{E}} \cap(F, E)_{e_{H}}=\phi$ and $e_{G_{E}} \subseteq(G, E)$ and $e_{H_{E}} \cap(G, E)=\phi$.It follows that, $\cup_{e_{H} \in\left(e_{G}\right)^{c}(F, E)_{e_{H}} \subseteq e_{G E}^{c}}$ Now, we want to prove that $e_{G_{E}^{c}}^{c} \subseteq$ $\cup_{e_{H} \in\left(e_{G}\right)^{c}}(F, E)_{e_{H}}$.Let $\quad \cup_{e_{H} \in\left(e_{G}\right)^{c}}(F, E)_{e_{H}}=(H, E)$. Where $\quad H(e)=$ $\cup_{e_{H} \in\left(e_{G}\right)^{c}\left(F(e)_{e_{H}}\right.}$ for all $e \in E$.Since $e_{G_{E}^{c}}(e)=\left(e_{G}\right)^{c}$ for all $e \in E$ from Definition 9, so, for all $e_{H} \in\left\{e_{G}\right\}^{c}$ and $\quad e \in E \quad e_{G_{E}^{c}}^{c}(e)=\left\{e_{G}\right\}^{c}=$ $\cup_{e_{H} \in\left(e_{G}\right)^{c}}\left\{e_{H}\right\}=\cup_{e_{H} \in\left(e_{G}\right)^{c} F(e)_{e_{H}}=H(e)}$.Thus, $e_{G_{E}^{c}}^{c} \subseteq \cup_{e_{H} \in\left(e_{G}\right)^{c}}(F, E)_{e_{H}}$ from Definition 2, and so, $e_{G_{E}}^{c}=\mathrm{U}_{e_{H} \in\left(e_{G}\right)^{c}}(F, E)_{e_{H}}$.

This means that, $e_{G_{E}}^{c}$ is soft open set for all $e_{H} \in\left\{e_{G}\right\}^{c}$.Therefore, $e_{G_{E}}$ is closed soft set.

Proposition 15. Let $\left(X, \tau_{1}, \tau_{2}, \tau_{3}, \tau_{4}, E\right)$ be a soft quad topological space over X. Then, if $\left(X, \tau_{1}, \tau_{2}, E\right)$ and $\left(X, \tau_{3}, \tau_{4}, E\right)$ are soft $T_{3}$ space, then $\left(X, \tau_{1}, \tau_{2}, \tau_{3}, \tau_{4}, E\right)$ is a pair wise soft $T_{2}$ space.

Proof: Suppose if $\left(X, \tau_{1}, \tau_{2}, E\right)$ is a soft $T_{3}$ space with respect to $\left(X, \tau_{3}, \tau_{4}, E\right)$, then according to definition for, $e_{G} \neq e_{H}, e_{G}, e_{H} \in X_{A}$, by using Theorem $8,\left(e_{H}, \mathrm{E}\right)$ is soft closed set in $\left(X, \tau_{3}, \tau_{4}, E\right)$ and $e_{G} \notin\left(e_{H}, E\right)$ there exist a $\left(X, \tau_{1}, \tau_{2}, E\right)$ soft open set $(F, E)$ and a $\left(X, \tau_{3}, \tau_{4}, E\right)$ soft open set $(G, E)$ such that $e_{G} \in(F, E), e_{H} \in(y, E) \subseteq(G, E)$ and $\left(F_{1}, E\right) \cap\left(F_{2}, E\right)=$ $\phi$. Hence, $\left(X, \tau_{1}, \tau_{2}, E\right)$ is soft $T_{2}$ space with respect to $\left(X, \tau_{3}, \tau_{4}, E\right)$ Similarly, if $\left(X, \tau_{3}, \tau_{4}, E\right)$ is a soft $T_{3}$ space with respect to $\left(X, \tau_{1}, \tau_{2}, E\right)$, then according to definition for , $e_{G} \neq e_{H}, e_{G}, e_{H} \in X_{A}$, by using Theorem 8, $\left(e_{G}, \mathrm{E}\right)$ is closed soft set in $\left(X, \tau_{1}, \tau_{2}, E\right)$ is and $y \notin(x, E)$ there exists a $\left(X, \tau_{3}, \tau_{4}, E\right)$ soft open set $(F, E)$ and a $\left(X, \tau_{1}, \tau_{2}, E\right)$ soft open set $(G, E)$ such that $e_{H} \in(F, E), \quad e_{G} \in(x, E) \subseteq(G, E)$ and $\left(F_{1}, E\right) \cap\left(F_{2}, E\right)=\phi$. Hence, $\left(X, \tau_{3}, \tau_{4}, E\right)$ is a soft $T_{2}$ space. Thus $\left(X, \tau_{1}, \tau_{2}, \tau_{3}, \tau_{4}, E\right)$ is a pair wise soft $T_{2}$ space.

Proposition 16. Let $\left(X, \tau_{1}, \tau_{2}, \tau_{3}, \tau_{4}, E\right)$ be a soft quad topological space over $X$. If if $\left(X, \tau_{1}, \tau_{2}, E\right)$ and $\left(X, \tau_{3}, \tau_{4}, E\right)$ are soft $T_{3}$ space then $\left(X, \tau_{1}, \tau_{2}, \tau_{3}, \tau_{4}, E\right)$ is a pair wise soft $T_{3}$ space.

Proof: Suppose $\left(X, \tau_{1}, \tau_{2}, E\right)$ is a soft $T_{3}$ space with respect to $\left(X, \tau_{3}, \tau_{4}, E\right)$ then according to definition for $e_{G}, e_{H} \in X_{A} e_{G} \neq e_{H}$ there happens $\tau_{1} \cup \tau_{2}$ soft open set $(F, E)$ and a $\tau_{3} \cup \tau_{4}$ soft open set $(G, E)$ such that $e_{G} \in(F, E)$ and $e_{H} \notin(F, E)$ or $e_{H} \in(G, E)$ and $e_{G} \notin(G, E)$ and for each point $e_{G} \in X_{A}$ and each $\tau_{1} \cup \tau_{2}$ closed soft set $\left(G_{1}, E\right)$ such that $e_{G} \notin$ $\left(G_{1}, E\right)$ there happens a $\tau_{1} \cup \tau_{2}$ soft open set $\left(F_{1}, E\right)$ and $\tau_{3} \cup \tau_{4}$ soft open set $\left(F_{2}, E\right)$ such that $e_{G} \in\left(F_{1}, E\right),\left(G_{1}, E\right) \subseteq\left(F_{2}, E\right)$ and $\left(F_{1}, E\right) \cap\left(F_{2}, E\right)=$ $\phi$. Similarly $\left(X, \tau_{3}, \tau_{4}, E\right)$ is a soft $T_{3}$ space with respect to $\left(X, \tau_{1}, E\right)$. So according to definition for $e_{G}, e_{H} \in X_{A}, e_{G} \neq e_{H}$ there exists a $\tau_{3} \cup \tau_{4}$ soft open set $(F, E)$ and $\tau_{1} \cup \tau_{2}$ soft open set $(G, E)$ such that $e_{H} \in(F, E)$ and $e_{H} \notin(F, E)$ or $e_{H} \in(G, E)$ and $e_{G} \notin(G, E)$ and for each point $e_{G} \in X_{A}$ and each $\tau_{3} \cup \tau_{4}$ closed soft set $\left(G_{1}, E\right)$ such that $e_{G} \notin\left(G_{1}, E\right)$ there exists $\tau_{3} \cup$ 
$\tau_{4}$ soft open set $\left(F_{1}, E\right)$ and $\tau_{1} \cup \tau_{2}$ soft open set $\left(F_{2}, E\right)$ such that $e_{G} \in$ $\left(F_{1}, E\right),\left(G_{1}, E\right) \subseteq\left(F_{2}, E\right) \quad$ and $\quad\left(F_{1}, E\right) \cap\left(F_{2}, E\right)=\phi . \quad$ Hence $\left(X, \tau_{1}, \tau_{2} \tau_{3}, \tau_{4}, E\right)$ is pair wise soft $T_{3}$ space.

Proposition 17. If $\left(X, \tau_{1}, \tau_{2} \tau_{3}, \tau_{4}, E\right)$ be a soft quad topological space over $\mathrm{X}$. $\left(X, \tau_{1}, \tau_{2}, E\right)$ and $\left(X, \tau_{3}, \tau_{4}, E\right)$ are soft $T_{4}$ space then $\left(X, \tau_{1}, \tau_{2}, \tau_{3}, \tau_{4}, E\right)$ is pair wise soft $T_{4}$ space.

Proof: Suppose $\left(\left(X, \tau_{1}, \tau_{2}, E\right)\right)$ is soft $T_{4}$ space with respect to $\left(X, \tau_{3}, \tau_{4}, E\right)$.So according to definition for $e_{G}, e_{H} \in X_{A}, e_{G} \neq e_{H}$ there happens a $\tau_{1} \cup \tau_{2} \quad$ soft open set $(F, E)$ and a $\tau_{3} \cup \tau_{4}$ soft open set $(G, E)$ such that $e_{G} \in(F, E)$ and $e_{H} \notin(F, E)$ or $e_{H} \in(G, E)$ and $e_{G} \notin(G, E)$ each $\tau_{1} \cup \tau_{2}$ soft closed set $\left(F_{1}, E\right)$ and a $\tau_{3} \cup \tau_{4}$ soft closed set $\left(F_{2}, E\right)$ such that $\left(F_{1}, E\right) \cap\left(F_{2}, E\right)=\phi$. There occurs $\left(F_{3}, E\right)$ and $\left(G_{1}, E\right)$ such that $\left(F_{3}, E\right)$ is soft $\tau_{3} \cup \tau_{4}$ open set $\left(G_{1}, E\right)$ is soft a $\tau_{1} \cup \tau_{2}$ open set $\left(F_{1}, E\right) \subseteq$ $\left(F_{3}, E\right),\left(F_{2}, E\right) \subseteq\left(G_{1}, E\right)$ and $\left(F_{3}, E\right) \cap\left(G_{1}, E\right)=\phi$. Similarly, $\tau_{3} \cup \tau_{4}$ is soft $T_{4}$ space with respect to $\tau_{1} \cup \tau_{2}$ so according to definition for $e_{G}, e_{H} \in$ $X_{A}, e_{G} \neq e_{H}$ there happens a $\tau_{3} \cup \tau_{4}$ soft $\beta$ open set $(F, E)$ and a $\tau_{1} \cup$ $\tau_{2}$ soft $\beta$ open set $(G, E)$ such that $e_{G} \in(F, E)$ and $e_{H} \notin(F, E)$ or $e_{H} \in$ $(G, E)$ and $e_{G} \notin(G, E)$ and for each $\tau_{3} \cup \tau_{4}$ soft closed set $\left(F_{1}, E\right)$ and $\tau_{1} \cup$ $\tau_{2}$ soft closed set $\left(F_{2}, E\right)$ such that $\left(F_{1}, E\right) \cap\left(F_{2}, E\right)=\phi$.there occurs $\left(F_{3}, E\right)$ and $\left(G_{1}, E\right)$ such that $\left(F_{3}, E\right)$ is soft $\tau_{3} \cup \tau_{4}$ open set $\left(G_{1}, E\right)$ is soft $\tau_{1} \cup$ $\tau_{2}$ open set such that $\left(F_{1}, E\right) \subseteq\left(F_{3}, E\right),\left(F_{2}, E\right) \subseteq\left(G_{1}, E\right)$ and $\left(F_{3}, E\right) \cap$ $\left(G_{1}, E\right)=\phi$ hence $\left(X, \tau_{1}, \tau_{2}, \tau_{3}, \tau_{4}, E\right)$ is pair wise soft $T_{4}$ space.

Proposition 18. Let $\left(X, \tau_{1}, \tau_{2}, \tau_{3}, \tau_{4}, E\right)$ be a soft quad topological space over $X$ and $Y$ be a non-empty subset of $X$. if $\left(Y, \tau_{1 Y}, \tau_{2 Y}, \tau_{3 Y}, \tau_{4 Y}, E\right)$ is pair wise soft $T_{3}$ space. Then $\left(Y, \tau_{1 Y}, \tau_{2 Y}, \tau_{3 Y}, \tau_{4 Y}, E\right)$ is pair wise soft $T_{3}$ space.

Proof. First we prove that $\left(Y, \tau_{1 Y}, \tau_{2 Y}, \tau_{3 Y}, \tau_{4 Y}, E\right)$ is pair wise soft $T_{1}$ space. Let $e_{G}, e_{H} \in X_{A}, e_{G} \neq e_{H}$ if $\left(X, \tau_{1}, \tau_{2}, \tau_{3}, \tau_{4}, E\right)$ is pair wise soft space then this implies that $\left(X, \tau_{1}, \tau_{2}, \tau_{3}, \tau_{4}, E\right)$ is pair wise soft $\tau_{1} \cup \tau_{2}$ space. So there exists $\tau_{1} \cup \tau_{2}$ soft open set $(G, E)$ such that $e_{G} \in(F, E)$ and $e_{H} \notin(F, E)$ or $e_{H} \in(G, E)$ and $e_{G} \notin(G, E)$ now $e_{G} \in Y$ and $e_{G} \notin(G, E)$. Hence $e_{G} \in Y \cap$ $(F, E)=\left(Y_{F}, E\right)$ then $e_{H} \notin Y \cap F(\alpha)$ for some $\alpha \in E$. this means that $\alpha \in E$ then $e_{H} \notin Y \cap F(\alpha)$ for some $\alpha \in E$.

There fore, $e_{H} \notin Y \cap(F, E)=\left(Y_{F}, E\right)$. Now $e_{H} \in Y$ and $e_{H} \in(G, E)$. Hence, $e_{H} \in Y \cap(G, E)=\left(G_{Y}, E\right)$ where $(G, E) \in \tau_{3} \cup \tau_{4} \quad$. Consider $x \notin(G, E)$ .this means that $\alpha \in E$ then $x \notin Y \cap G(\alpha)$ for some $\alpha \in E$. There fore $e_{G} \notin$ $Y \cap(G, E)=\left(G_{Y}, E\right)$ thus $\left(Y, \tau_{1 Y}, \tau_{2 Y}, \tau_{3 Y}, \tau_{4 Y}, E\right)$ is pair wise soft $T_{1}$ space Now, we prove that $\left(Y, \tau_{1 Y}, \tau_{2 Y}, \tau_{3 Y}, \tau_{4 Y}, E\right)$ is pair wise soft $T_{3}$ space.

Let $e_{H} \in Y$ and $(G, E)$ be soft closed set in Y such that $e_{H} \notin(G, E)$ where $(G, E) \in \tau_{1} \cup \tau_{2}$ then $(G, E)=(Y, E) \cap(F, E)$ for some soft closed set in $\tau_{1} \cup \tau_{2}$ hence $e_{H} \notin(Y, E) \cap(F, E)$ but $e_{H} \in(Y, E)$, so $e_{H} \notin(F, E)$ since $\left(X, \tau_{1}, \tau_{2}, \tau_{3}, \tau_{4}, E\right)$ is soft $T_{3}$ space

$\left(X, \tau_{1}, \tau_{2}, \tau_{3}, \tau_{4}, E\right)$ is soft regular space so there happens $\tau_{1} \cup \tau_{2}$ soft open set $\left(F_{1}, E\right)$ and $\tau_{3} \cup \tau_{4}$ soft open set $\left(F_{2}, E\right)$ such that

$$
e_{H} \in\left(F_{1}, E\right),(G, E) \subseteq\left(F_{2}, E\right)
$$

$$
\left(F_{1}, E\right)\left(F_{2}, E\right)=\phi
$$

Take $\left(G_{1}, E\right)=(Y, E) \cap\left(F_{2}, E\right)$ then $\left(G_{1}, E\right),\left(G_{2}, E\right)$ are soft open sets in Y such that

$$
\begin{aligned}
& e_{H} \in\left(G_{1}, E\right),(G, E) \subseteq(Y, E) \cap\left(F_{2}, E\right) \\
& =\left(G_{2}, E\right) \\
& \left(G_{1}, E\right) \cap\left(G_{2}, E\right) \subseteq\left(F_{1}, E\right) \cap\left(F_{2}, E\right)=\phi \\
& \left(G_{1}, E\right) \cap\left(G_{2}, E\right)=\phi
\end{aligned}
$$

Therefore, $\left(\tau_{1 Y}, \tau_{2 Y}\right)$ is soft regular space with respect to $\left(\tau_{3 Y}, \tau_{4 Y}\right)$ Similarly, Let $e_{H} \in Y$ and $(G, E)$ be a soft closed sub set in Y such that $e_{H} \notin$ $(G, E)$, Where $(G, E) \in \tau_{3} \cup \tau_{4}$ then $(G, E)=(Y, E) \cap(F, E)$ where $(F, E)$ is some soft closed set in $\tau_{3} \cup \tau_{4} . e_{H} \notin(Y, E) \cap(F, E)$ But $e_{H} \in(Y, E)$ so $e_{H} \notin$ $(F, E)$ since $\left(X, \tau_{1}, \tau_{2}, \tau_{3}, \tau_{4}, E\right)$ is soft regular space so there happens $\tau_{3} \cup \tau_{4}$ soft open set $\left(F_{1}, E\right)$ and $\tau_{1} \cup \tau_{2} \operatorname{soft}$ open $\operatorname{set}\left(F_{2}, E\right)$. Such that

$$
e_{H} \in\left(F_{1}, E\right),(G, E) \subseteq\left(F_{2}, E\right)
$$$$
\left(F_{1}, E\right) \cap\left(F_{2}, E\right)=\phi
$$

Take $\quad\left(G_{1}, E\right)=(Y, E) \cap\left(F_{1}, E\right)$

$$
\left(G_{1}, E\right)=(Y, E) \cap\left(F_{1}, E\right)
$$

Then $\left(G_{1}, E\right)$ and $\left(G_{2}, E\right)$ are soft open set in Y such that

$$
e_{H} \in\left(G_{1}, E\right),(G, E) \subseteq(Y, E) \cap\left(F_{2}, E\right)=
$$

Therefore $\left(\tau_{3 Y}, \tau_{4 Y}\right)$ is soft regular space.

$$
\left(G_{1}, E\right) \cap\left(G_{2}, E\right) \subseteq\left(F_{1}, E\right) \cap\left(F_{2}, E\right)=\phi
$$

\section{CONCLUSION}

A soft set with single specific topological structure is unable to accept the duty to construct the whole theory. So to make the theory rich, some superfluous structures on soft set has to be announced. It makes, it more springy to develop the soft topological spaces with its countless applications. In this respect we introduce strong topological structure known as soft quad topological structure in this article.
Topology is such supreme branch of mathematics which catches the applications of both pure and applied mathematics in an attractive way. When mathematicians talk about structures then the concept of soft topology automatically come in action. Recently, many scholars have studied the soft set theory which is coined by Molodtsov [16] and carefully applied to many difficulties which contain uncertainties in our social life. Shabir and Naz [18] familiarized and deeply studied the origin of soft topological spaces. They also studied topological structures and exhibited their several properties with respect to ordinary points.

In the present work, we continuously study the character of soft separation axioms in quad soft topological spaces with respect to soft points as well as ordinary points of a soft topological space. We introduce soft $q T_{0}$ structure, soft $q T_{1}$ structure, soft $q T_{2}$ structure, soft $q T_{3}$ and soft $q T_{4}$ structure with respect to soft and crisp points

\section{REFERENCES}

[1] Zadeh, L.A. 1965. Fuzzy sets. Information and Control, 8, 338-353.

[2] Atanassov, K. 1986. Intuitionistic fuzzy sets. Fuzzy Sets and Systems, 20, 87-96.

[3] Pawlak, Z. 1982. Rough sets. Journal of Information and Computing Science, 11, 341-356.

[4] Molodtsov, D. 1999. Soft set theory-first results. Computers \& Mathematics with Applications, 37, 19-31.

[5] Maji, P.K., Roy, A.R., Biswas, R. 2002. An Application of soft sets in a decision-making problem. Computers \& Mathematics with Applications, 44, 1077-1083.

[6] Aktas, H., Cagman, N. 2007. Soft sets and soft group. Information Science, 177, 2726-2735.

[7] Acar, U., Koyuncu, F., Tanay, B. 2010. Soft sets and soft rings. Computers \& Mathematics with Applications, 59 3458-3463.

[8] Feng, F., Jun, Y.B., Zhao, X. 2008. Soft semi rings. Computers \& Mathematics with Applications, 56 (10), 2621-2628.

[9] Sun, Q.M., Zhang, Z.L., Liu, J. 2008. Soft sets and soft modules. Lecture Notes in Computer Science, 5009 (2), 403-409.

[10] Shabir, M., Ali, M.I. 2009. Soft ideals and generalized fuzzy ideals in semi groups. New Mathematics and Natural Computation, 5, 599-615.

[11] Aras, C.G., Bayramov, S. 2011. Intuitionistic fuzzy soft modules. Computers and Mathematics with Applications, 62, 2480-2486.

[12] Aras, C.G., Bayramov, S. 2011. Fuzzy soft modules. International Mathematical Forum, 6 (11), 517-527.

[13] Kandil, A., Tantawy, O.A.E., El-Sheikh, S.A., Hazza, S.A. 2016. Pairwise open (closed) soft sets in soft bi topological spaces. Annals of Fuzzy Mathematics and Informatics, 11 (4), 571-588.

[14] Cagman, N., Karatas, S., Enginoglu, S. 2011. Soft topology. Computers \& Mathematics with Applications, 62, 351-358.

[15] Min, W.K. 2011. A note on soft topological spaces. Computers \& Mathematics with Applications, 62, 3524-3528.

[16] Sabir, H., Bashir, A. 2011. Some properties of soft topological spaces. Computers \& Mathematics with Applications, 62 (13), 4058-4067.

[17] Zorlutana, I., Akdag, M., Min, W.K., Atmaca, S. 2012. Remarks on soft topological spaces. Annals of Fuzzy Mathematics and Informatics, 3 (2), 171-185.14

[18] Mashhour, A.S., Allam, A.A., Mahmoud, F.S., Khedr, F.H. 1983. On supra topological spaces. Indian Journal of Pure and Applied Mathematics, 14 (4), 502-510.

[19] El-Sheikh, S.A., Abd El-latif, A.M. 2014. Decomposition of Some Types of Supra Soft Sets and Soft Continuity. International Journal of Mathematics Trends and Technology, 9 (1), 37-56.

[20] Ittanagi, B.M. 2014. Soft bi topological spaces. International Journal of Computer Applications, 107 (7), 1-4 
[21] Ali, M.I., Feng, F., Liu, X.Y., Min, W.K., Shabir, M. 2009. On some new operations in soft set theory. Computers \& Mathematics with Applications, $57,1547-1553$

[22] Shabir, M., Naz, M. 2011. On soft topological spaces. Computers \& Mathematics with Applications, 61, 1786-1799.

[23] Hussain, S., Ahmad, B. 2015. Soft separation axioms in soft topological space submitted to Elsevier 28 September.

[24] Bayramov, S., Gunduz, C. 2013. Soft locally compact spaces and soft paracompact spaces. International Journal of Mathematics and Systems Science, 3, 122-130. 